\title{
3-dimensional water display
}

\author{
Aleksandr Chekhovski ${ }^{1,2 a)}$ and Hiroshi Toshiyoshi ${ }^{1,2}$ \\ ${ }^{1}$ Kanagawa Academy of Science and Technology, \\ 3-2-1 Sakado, Takatsu-ku, Kawasaki, Kanagawa 213-0012, Japan \\ ${ }^{2}$ Institute of Industrial Science, the University of Tokyo, \\ 4-6-1 Komaba, Meguro-ku, Tokyo 153-8505, Japan \\ a)alex@iis.u-tokyo.ac.jp
}

\begin{abstract}
A displaying technique of real 3D dynamic images inside a transparent liquid is demonstrated for the first time. Images were formed by micro-flashes generated by means of laser breakdown in liquid. Amongst several types of liquid tested, tap water was found to be an optimal medium for the displaying due to its lowest breakdown threshold power. Electronic color filter was exploited for dynamic coloring of flashes in synchronization with 3D steering of laser beam's focal point.
\end{abstract}

Keywords: 3D display, dynamic, liquid, laser, breakdown, color

Classification: Photonics devices, circuits, and systems

\section{References}

[1] W.-S. Chun, J. Napoli, O. S. Cossairt, R. K. Dorval, D. M. Hall, T. J. Purtell II, J. F. Schooler, Y. Banker, and G. E. Favalora, "Spatial 3D Infrastructure: Display-Independent Software Framework, High-Speed Rendering Electronics, and Several New Displays," Proc. SPIE, vol. 5664, pp. 302-312, 2005.

[2] B. G. Blundell and A. J. Schwarz, Volumetric Three Dimensional Display Systems, New York: John Wiley\&Sons, 2000.

[3] M. E. Motamedi, "Digital micromirror device," in MOEMS, pp. 400-408, SPIE, Bellingham, 2005.

[4] E. Downing, L. Hesselink, J. Ralston, and R. Macfarlane, "A Three-Color Solid-State Three-Dimensional Display," Science, vol. 273, pp. 1185-1189, 1996.

[5] Laser breakdown 3D engraving in glass technology [Online] http://www.crystalimpressions.co.uk/

[6] H. Kimura, T. Uchiyama, and H. Yoshikawa, "Laser produced 3D display in the air," Proc. of Int. Conf. on Comp. Graphics and Interactive Tech. (ACM SIGGRAPH 2006 Emerging technologies) Boston, USA, Article, no. 20, 2006.

[7] D. A. Glazer, "Bubble Chamber," Nobel Prize in Physics, 1960.

[8] P. K. Kennedy, D. X. Hammer, and B. D. Rocwell, "Laser induced breakdown in aqueous media," Progr. Quant. Electr., vol. 21, pp. 155-248, 1997. 


\section{Introduction}

Three-dimensional (3D) image displays using free space have been a longcherished dream ever since the beginning of the 20th century, but no commercial products have been put in the consumer electronics market due to technical difficulties, except for a spinning screen type [1]. For instance, stereoscopic 3D display produces discomfort to viewer's eyes, and holographic 3D display is extremely complex in data computation [2]. In contrast to these, use of electro-mechanical optical scanners [3] for spatial light modulation is promising to create real 3D images that could be recognized by viewers without forcing discomfort. Optical scanners and lasers have been used to creating of 3D images in a transparent solid $[4,5]$ or gaseous medium [6] but no liquid medium has been exploited for 3D imaging ever, except for the bubble chamber [7]. In this paper, we demonstrate for the first time a displaying of real 3D dynamic colored images inside a transparent liquid (water) using the optical scanners and laser breakdown [8] micro-flashes as 3D pixels or called voxels.

\section{Experiments}

In our experiment, we used an invisible beam of YAG:Nd Q-switched laser (1064 nm of wavelength, $4 \mathrm{~mJ} / 20 \mathrm{~ns}$ of $\max$ pulse power, $5 \mathrm{kHz}$ of $\max$ pulsing, $2 \mathrm{~mm}$ of beam diameter). Three-dimensional beam steering was made by using a glass lens on an electromechanical shuttle for the longitudinal $\mathrm{Z}$-direction and also by using two mirror-scanners for the transversal $\mathrm{X}$ and $\mathrm{Y}$ directions.

The breakdown flash was accompanied with satellite bubble, as shown in Fig. 1. The flashing light was found to be more visible than the reflection from the bubble and it looked to be a white shining dot, which could be observed even by naked eyes from a 5-meter-distance in the indoor lighting.

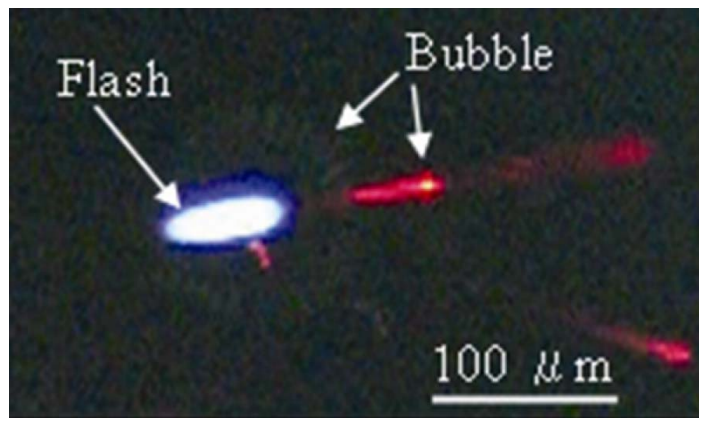

Fig. 1. Laser breakdown in water photographed at $1 / 30 \mathrm{~s}$ resolution with red lighting. The laser beam impinged the water from the left-hand side of the photo, and a flash and a bubble were formed at the focal point. The bubble around flash was difficult to see due to its short lifetime. Breakdown plasma flash looked more visible than the satellite bubbles. 
Three-dimensional Lissajous figures of approximately $1 \mathrm{~cm}$ in size were drawn by the series of flashing dots, in a container filled with water, as shown in Fig. 2. Upwards of 500 laser pulses per second were applied to a $1 \mathrm{~cm}^{3}$ volume of water to create the dynamic 3D figures for several tens of minutes. Satellite bubbles generated in conjunction with the flashes did not seem to hamper the 3D image.

It was found to be impossible to keep generating flashes in water by immovable laser beam focused in the same spot of water at laser pulsing of more than $500 \mathrm{~Hz}$. Hence, spatial resolution and time resolution of the displaying in water can be evaluated as high as 10 pixels per millimeter and 500 frames per second accordingly. These resolution limits are sufficient for the displaying of a high-quality dynamic image.

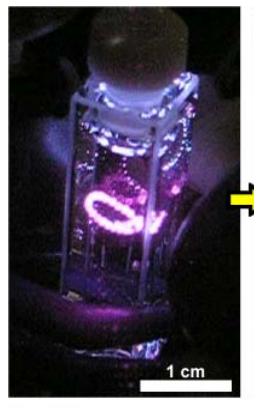

$0 \mathrm{~s}$

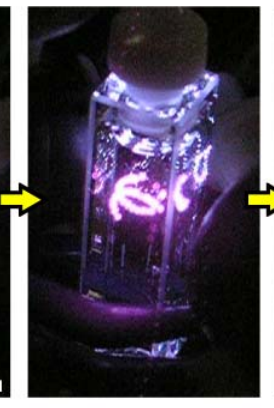

$1 / 30 \mathrm{~s}$

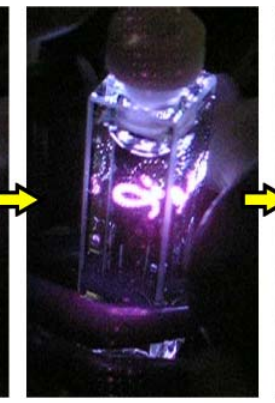

$2 / 30 s$

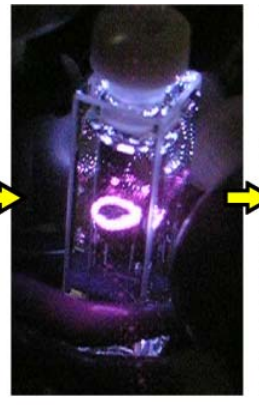

$3 / 30 \mathrm{~s}$

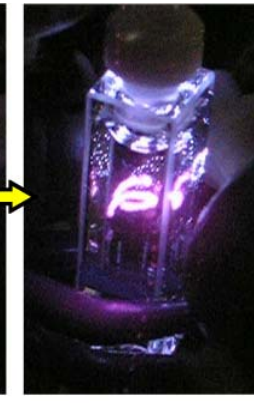

$4 / 30 \mathrm{~s}$

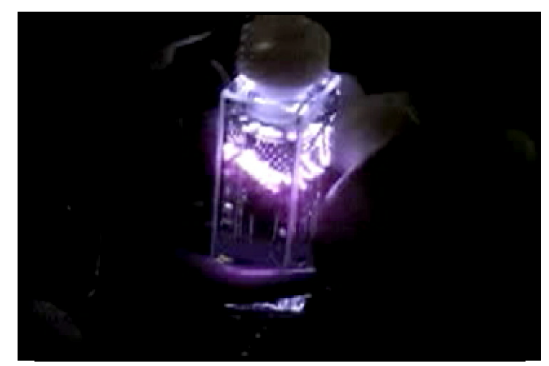

Fig. 2. A series of snapshots showing a running real 3D shining spiral in a transparent container filled with tap water. The spiral seemed to be chopped due to the shutter speed of the video camera. See a movie file attached.

The breakdown in liquid generated also a sparking sound at the frequency of the laser pulsing; the sound could be eventually eliminated or suppressed by using pulsing frequency in ultrasound range (up to $10^{9} \mathrm{~Hz}$ for femtosecond lasers).

Similar 3D display has been reported by Kimura et al. [5] for air plasma, but they required high power laser of $250 \mathrm{~mJ} / 7 \mathrm{~ns}$. High power laser can not provide the high repetition rate which is necessary for the dynamic imaging. In contrast to this, breakdown imaging in water needed only $4 \mathrm{~mJ} / 20 \mathrm{~ns}$ for laser pulse. For comparison, with our laser, it was necessary to use an aspherical lens with focal distance of $5 \mathrm{~mm}$ or less in order to execute laser 
breakdown in air, while laser breakdown in tap water was executed with a usual biconvex lens of $50 \mathrm{~mm}$ focal distance. Further lowering the breakdown threshold can open up a door to the 3D images of large display size with small lasers. Until today, we found that the laser breakdown threshold was lowest in tap water, compared aqueous solutions containing such as $\mathrm{KI}+\mathrm{I}_{2}$, emulsion of $\mathrm{CuI}$ or $\mathrm{CuCl}$.

Optical spectrum of the light flash covers the whole visible wavelength range. Therefore an electronic color filter can be used for coloring the flash light spot in any color (Fig. 3). In our experiments, we used a TFT (thinfilm transistor)-driven color filter of an LCD (liquid-crystal display) to change colors in synchronization with the laser pulse and the scanning system.
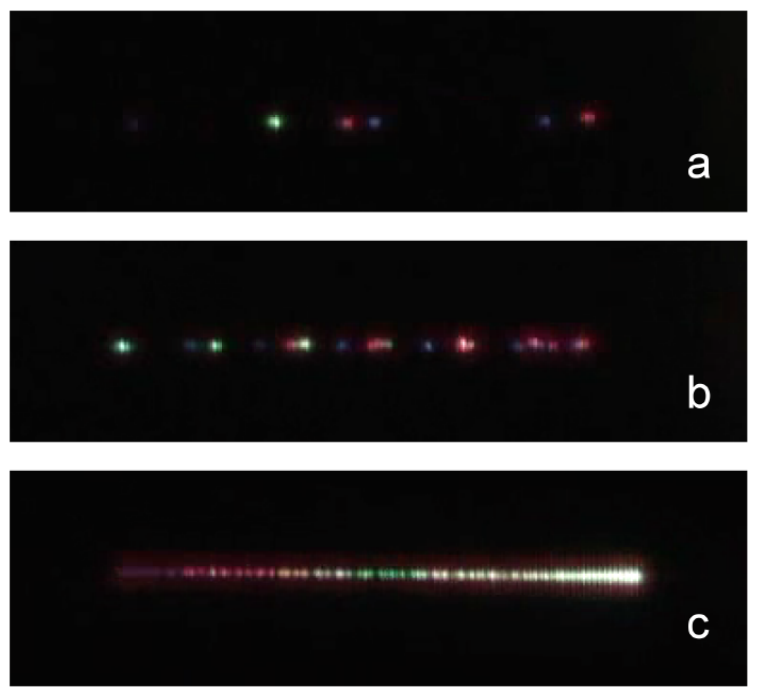

Fig. 3. Snapshots of color-filtered flashing dot series generated with different laser pulsing of (a) $10 \mathrm{~Hz}$, (b) $50 \mathrm{~Hz}$, (c) and $300 \mathrm{~Hz}$. Shutter speed was set to be one second. The container with tap water was placed behind the color filter (TFT matrix of LCD), changing its colors quickly between blue, red, yellow and green during the longitudinal steering for a distance of $4 \mathrm{~cm}$.

\section{Conclusion}

In conclusion, for the first time a liquid medium was investigated for the creation of real 3D dynamic color images. It was shown experimentally, that the breakdown micro-flash in liquid could be used as voxel of white color. Seeing through electronic driven color filter made a voxel to be perceived like colored one. Tap water was found to be an optimal medium for the displaying due to the lowest breakdown threshold. Our next step is to create arbitrary images by PC controlling the laser, scanners and the color filter. 


\section{Acknowledgements}

We thank Prof. Kazuhiko Hirakawa and Dr. Yukiko Otsuka with the Institute of Industrial Science, the University of Tokyo for their advice and technical assistance for using their high-Q laser. We also would like to thank Photron

Limited for their providing us with their high-speed camera to observe the laser breakdown flashes. 